\title{
WATER ADSORPTION IN HYDROPHILIC ZEOLITES: EXPERIMENT AND SIMULATION
}

\author{
$\underline{\text { Juan Manuel Castillo }}^{1}$, Joaquín Silvestre Albero ${ }^{2}$, Francisco Rodriguez-Reinoso ${ }^{2}$, \\ Thijs J.H. Vlugt ${ }^{3}$, and Sofia Calero ${ }^{4}$ \\ ${ }^{1}$ Laboratory of Engineering Thermodynamics, Erwin-Schrödinger-straße 44, \\ University of Kaiserslautern, 67663Kaiserslautern, Germany \\ ${ }^{2}$ Laboratorio de Materiales Avanzados, Departamento de Quimica Inorgánica, Universidad de Alicante, \\ Ctra. San Vicente-Alicante s/n,Ap. 99, E- 03080 Alicante, Spain \\ ${ }^{3}$ Department of Process and Energy, Delft University of Technology, \\ Leeghwaterstraat 44, 2628CA Delft, The Netherlands \\ ${ }^{4}$ Faculty of Experimental Sciences, University Pablo de Olavide, Ctra. de Utrera km. 1, 41013 Sevilla, Spain
}

We have measured experimental adsorption isotherms of water in zeolite LTA4A, and studied the regeneration process by performing subsequent adsorption cycles after degassing at different temperatures. We observed incomplete desorption at low temperatures, and cation rearrangement at successive adsorption cycles. We also developed a new molecular simulation force field able to reproduce experimental adsorption isotherms in the range of temperatures between $273 \mathrm{~K}$ and 374 K. Small deviations observed at high pressures are attributed to the change of the water dipole moment at high loadings. The force field correctly describes the preferential adsorption siting of water at different pressures. We tested the influence of the zeolite structure, framework flexibility, and cation mobility when considering adsorption and diffusion of water. Finally, we performed checks on force field transferability between different hydrophilic zeolite types, concluding that classical, non-polarizable water force fields are not transferable. 


\section{Introduction}

The adsorption of water in hydrophilic zeolites is an important process in the purification of wastewater, catalysis, and gas separation. In particular, zeolite LTA4A is widely used to separate polar from non-polar molecules by permeation, as this zeolite is highly hydrophilic due to its low $\mathrm{Si} / \mathrm{Al}$ ratio $^{1}$. For example, LTA4A has proven to be useful in alcohol dehydratation ${ }^{2-4}$, pollutant removal from water ${ }^{5,6}$, or even as working medium in the refrigeration of thermal machines ${ }^{7}$.

Experimental adsorption isotherms of water in zeolites provided by different research groups often show large deviations ${ }^{8,9}$, mainly due to imperfections in the zeolite crystals, cation relocation, or indetermination in the location of the $\mathrm{Al}$ atoms in the zeolite structure ${ }^{10}$. Therefore, it is important to compare different experimental sets to make sure that the adsorption data reported is consistent and reproducible. Molecular simulation studies also reveal large differences in adsorption depending on the simulation parameters and the models employed. In a previous work ${ }^{11}$, we studied water adsorption in hydrophobic zeolites by molecular simulation. We showed that water adsorption was extremely sensitive to small changes in the force field parameters, the selection of water model, the position of the framework atoms, and the partial charges of the zeolite atoms. There is a large number of simulation studies on the adsorption of water in different hydrophilic zeolites, mostly in $\mathrm{FAU}^{12,13}$ and $\mathrm{MFI}^{14,15}$, but also in others such as $\mathrm{HEU}^{16}, \mathrm{GOO}^{17}$, or MOR ${ }^{18}$. Many of them focus on the description of the cation and water adsorption sites, which are not very sensitive to the force field parameters. It still unknown whether it is possible to develop a classical, general force field able to correctly describe the adsorption of water in any hydrophilic zeolite. Although some research groups have used a determined force field to study adsorption in different hydrophilic zeolites $^{19,20}$, it is not clear if these force fields can reproduce the adsorption isotherms for different zeolites at different conditions. It has been shown that the adsorption calculated by molecular simulation in zeolites with non framework cations depends on the specific location of the alumina atoms in the structure ${ }^{21}$, which is usually unknown. In the case of the LTA4A zeolite, which has a $\mathrm{Si} / \mathrm{Al}$ ratio equal to one, the location of the non-framework cations is well known. In this zeolite, the positions of the silicon and alumina atoms alternate following the Lowenstein's rule, which forbids the bonding of two alumina atoms by an oxygen atom.

Another reason to focus on this particular zeolite is that it has been extensively studied both experimentally ${ }^{1,9}$, and by molecular simulation. Furukawa et al. ${ }^{22}$ used a rigid model of LTA4A with fixed cations and blocked beta cages to study adsorption and diffusion of water, ethanol, and their mixtures. These authors found large water adsorption selectivity, and a larger diffusion of 
water compared to ethanol, which is unable to cross the 8-ring windows of the structure. Jaramillo et $a .^{23}$ studied the adsorption and adsorption siting as a function of loading of water and other small molecules in LTA4A, using a rigid model where the non-framework cations did not move from their crystallographic positions. Although not explicitly compared with experimental data, their water adsorption is too large, probably due to the large partial charges of the framework atoms. Kristof et al. ${ }^{24}$ simulated the adsorption of water, methanol, and their mixtures in LTA4A, showing the importance of water hydrogen bonding for adsorption. They found that adsorption takes place in two steps: a first layer close to the zeolite wall, and then a second layer on top of the first. In their simulations water molecules cannot enter the beta cages, because this is only allowed by translation moves and the diffusion through the 6-ring windows is very slow. Wu et al. ${ }^{25}$ studied the adsorption and diffusion of water/alcohol mixtures in LTA4A with a fully flexible zeolite model. They report adsorption with deviations of up to $10 \%$ respect to the experimental data, while the molecules are not able to enter the beta cages. Faux et al. ${ }^{26}$ perform Molecular Dynamics (MD) simulations to describe molecular siting. These authors conclude that there are four water molecules adsorbed in every beta cage, that the diffusion through the 6-rings is very slow, and that the water structure is very similar to the bulk water structure at short distances. Gren et al. ${ }^{27}$ also use MD simulations with a polarizable model for water, zeolite, and cations, to study the adsorption on the external surface of LTA4A. They observe adsorption by layers, and the leaching of the cations out of the zeolite. A similar approach was used by Allen et al. ${ }^{28}$ to study the adsorption and diffusion of water on the external surface of LTA4A, which was modeled with terminal silanol groups. Higgings et $a l .{ }^{29}$ used energy minimization methods to study molecular siting, concluding that the beta cage is the most stable site for water adsorption.

The structure of zeolite LTA4A is shown in Figure 1. It consist of sodalite, or beta cages, joined to each other in a cubic arrangement by 4 membered rings. The sodalite cages are accessible via 6 membered rings windows, only large enough to allow the diffusion of cations, water, and other few small atoms/molecules. The void space between sodalite cages is called alpha cage. Alpha cages are connected by 8 membered ring windows. The extra framework sodium cations of the zeolite are initially located in the dehydrated structure at three different adsorption sites: site I, at the center of the 6-rings; site II, on the plane of the 8-rings; site III, facing the 4-rings ${ }^{30}$.

Here we study water adsorption in the hydrophilic zeolite LTA4A, which has the advantage that the location of the framework atoms in the zeolite is unique and exactly known. We measure experimental adsorption isotherms, and create a new force field for guest-host interactions that can reproduce the experimental adsorption by molecular simulation. We test the influence of cation 
mobility in two different framework characterizations of LTA4A ${ }^{30,31}$, demonstrating the importance of cation rearrangement during adsorption. We check the ability of the new force field for describing water adsorption in different hydrophilic zeolites, and conclude that more refined models are needed to obtain a general force field for water adsorption in hydrophilic zeolites. Finally, we provide possible explanations to the deviation of our simulation results respect to the experiment at high pressures.

\section{Methods}

A commercial NaA zeolite supplied by Linde (zeolite Type A with LTA structure) has been used in this work. Zeolite $A$ has a typical unit cell composition of $\left(\mathrm{Na}_{12}\left[\mathrm{Al}_{12} \mathrm{Si}_{12} \mathrm{O}_{48}\right] \cdot 27 \mathrm{H}_{2} \mathrm{O}\right)_{8}$, with a $\mathrm{Si} / \mathrm{Al}$ ratio close to 1.0. The $\mathrm{NaA}$ zeolite crystals have an average size of $44 \mathrm{~nm}$, as deduced from XDR analysis. Water adsorption isotherms were performed in house made volumetric equipment (LMA-hydrosorb) equipped with one pressure transducer (1000 torr). Water adsorption isotherms were measured at $298 \mathrm{~K}$ and $334 \mathrm{~K}$ and up to $\mathrm{p} / \mathrm{p}_{0} \sim 1.0$ (water vapor pressure $=23.8$ torr and 156.5 torr, respectively). Before each adsorption measurement, zeolite samples were degassed at $573 \mathrm{~K}$ overnight under UHV conditions. Consecutive adsorption cycles were performed in order to analyze the regeneration of the zeolite NaA. A degassing treatment was performed in between cycles using different temperatures (423 K, $523 \mathrm{~K}$ and $573 \mathrm{~K}$ ) for $4 \mathrm{~h}$.

Adsorption isotherms were calculated by molecular simulations in the grand-canonical ensemble, where the temperature, volume and chemical potential of the system are constant ${ }^{32}$. The chemical potential is directly related to the fugacity, which can be computed from the pressure and a given equation of state. Here we will consider the ideal gas approximation for water, as the pressures considered were lower than $10 \mathrm{kPa}$. The insertion/deletion of molecules was performed using the Configurational-Bias Monte Carlo technique ${ }^{33}$, and the following Monte Carlo moves used for the water molecules: insertion/deletion of molecules (1/3 of the moves); regrow, translation, rotation, and random translation (1/6 of the moves for each type of move). Cation movement was achieved by translation and random translation movements (both movements with the same probability). The maximum translation and rotation distances were adjusted during the simulation to achieve an average acceptance probability of 50\%. Simulations were performed in cycles, every cycle containing a number of Monte Carlo moves equal to the number of molecules present in the system, with a minimum of $20^{34,35}$. We performed additional Molecular Dynamics simulations with mobile cations in the canonical ensemble to determine if there is diffusion through the windows accessing 
the beta cages ${ }^{36}$. The equations of movement were integrated using the Verlet algorithm with a time step of 0.5 fs during a minimum of 20 million production cycles. The temperature of the system was controlled with a Nosé-Hoover chain thermostat of length 3. The energy drift in the simulations was negligible.

We used the rigid Tip5pEw ${ }^{37}$ water model and an existent force field for the description of the zeolite $^{38}$. In a previous work ${ }^{11}$, we justified the use of this water model for studying the adsorption of water in zeolites. The water-zeolite and water-cation interactions were fitted to the experimental data obtained in this work. Two different characterizations of the LTA4A structure, hydrated and dehydrated, were used in the study to describe the atomic positions of the zeolite ${ }^{30,31}$. We used the characterizations of Fitch et al. ${ }^{39}$ and Olson ${ }^{40}$ to assign positions to the atoms of the FAU zeolite, and selected a random distribution of alumina atoms satisfying the Lowenstein's rule. The simulation box consisted of a single unit cell of zeolite. The cell was kept rigid in most calculations. In the simulations with flexible framework the bonded interactions were described by the Nicholas model $^{41}$. The model of Garcia-Sanchez et al. ${ }^{38}$ was used to assign point charges to the zeolite atoms. The extra framework cations that balance the net charge of the zeolite were initially placed at the crystallographic positions of their respective structure ${ }^{30,31}$, and either allowed to move, or left fixed. The Lennard-Jones interactions were truncated and shifted at a cut-off radius of $12 \AA$. Electrostatic interactions were calculated using the Ewald summation technique with a precision of $10^{-6} 42$. The non-bonded parameters of the force field used in this work are summarized in Table 1.

\section{Results and Discussion}

Figure 2 shows the experimental adsorption isotherms at $334 \mathrm{~K}$ for a different number of adsorption cycles (Figure 2 a-c), and degassing temperatures of 423 K, 523 K, and 573 K (Figure 2 d-f). The adsorption is clearly different between different cycles at the same degassing temperature, and at the same cycle and different degassing temperature. The adsorption is always lower in the first cycle, while in successive cycles the adsorption is basically the same (see Figure 2a-c). Larger differences occur at lower pressures, and in the case of the lowest degassing temperature (Figure 2a) there are clear deviations also at high pressures. This last effect is attributed to incomplete degassing, as it has been estimated that complete water desorption can only be achieved at temperatures larger than $423 \mathrm{~K}^{1}$, and some authors even suggest temperatures in the vicinity of 700 $\mathrm{K}^{43}$. Incomplete desorption can affect the isotherms at low pressures, as preadsorbed water molecules act as extra adsorption sites, increasing the adsorption capacity of the zeolite in the 
subsequent measurements. However, water adsorption will not be enhanced if these hydrated cations are not accessible, as it occurs in the first adsorption cycle. A different situation occurs when the pristine zeolite has been already exposed to water at saturation pressure, i.e. after the first cycle. Water adsorption can give rise to reallocation of the extra framework cations of the zeolite to more favorable positions, making them more accessible to water molecules and therefore increasing the adsorption capacity in subsequent cycles. It is well known that cations act as nucleation centers for water molecules, and that they reallocate in the presence of water ${ }^{44,45}$. In the rest of the study we will compare adsorption isotherms obtained by molecular simulation with the experimental adsorption isotherms obtained after several regeneration cycles, using a degassing temperature of $573 \mathrm{~K}$.

To test the validity of our experiments, we compare our adsorption isotherms to other experimental adsorption data taken from the literature at $298 \mathrm{~K}$ and $334 \mathrm{~K}$ (Figure 3 and 4 respectively). Our adsorption isotherms match the experimental isotherms of Gorbach et al. ${ }^{9}$ and Morris ${ }^{46}$ at both temperatures. Small deviations can be attributed to the fact that these isotherms were measured for the pelletized form of the zeolite, while we measured them in zeolite crystals. Although the effect of the binder is considered to be negligible due to its low surface area ${ }^{46}$, its effect in the adsorption is difficult to evaluate and it should not be completely ignored. The rest of the experimental data presented in Figs. 3 and $4^{8,47,48}$ deviates from the matching isotherms, and we will neglect them in the present study.

We fit the guest-host interactions of the force field to reproduce the experimental water adsorption isotherm in LTA4A at different temperatures, using mobile cations and the hydrated structure. The fitting procedure was a mixture of trial and error and the use of the downhill simplex algorithm ${ }^{49}$. The result is presented in Figure 5. Although our force field reproduces the general shape of the isotherm and the location of the condensation step in a large range of temperatures, it does not reproduce simultaneously the adsorption at low and high pressures. The interactions of water in a zeolite are different at high and low pressures. At low pressure, the water-zeolite interactions are dominant, while at high pressure the water-water interactions are more important ${ }^{50}$. This is also reflected in the fact that the structure of water at saturation conditions is very similar to the structure of bulk water ${ }^{26}$. The differences between simulation and experiment at high pressures can be attributed to the increase of the water dipole moment inside the zeolite when the loading increases. The importance of polarizability in the arrangement of adsorbed molecules in zeolites has been previously suggested ${ }^{51}$. Coudert et $a .^{19}$ demonstrated by Carr-Parrinello molecular dynamics simulations that the dipole moment of water molecules adsorbed in hydrophilic zeolites increases 
with loading, up to values similar to the dipole moment of bulk water. The Tip5pEw water model used in this study has a fixed dipole moment of $2.29 \mathrm{D}^{37}$, and therefore it does not take into account this effect. As the water dipole moment increases the adsorption increases, because the waterzeolite/water-cation interactions become stronger. This result was checked by some test simulations that artificially increased the dipole moment of water only at high pressure conditions by increasing the atomic partial charges of the water model. The calculated isotherm at $334 \mathrm{~K}$ perfectly matches the experimental isotherm at low pressures, while at higher pressures the adsorption is lower. At 1 $\mathrm{kPa}$, the deviation between the simulation and the experiment is of around $20 \%$. This large deviation disappears if we increase the water dipole to a value of $2.5 \mathrm{D}$ (Fig. 6). It is difficult to provide a more precise value due to the large error bars in the adsorption at high loadings. This larger value of the dipole moment is consistent with the dipole moment calculated for water at similar loadings in hydrophilic LTA-type zeolites, estimated in $2.9 \mathrm{D}^{19}$. Nevertheless, we do not have any guarantee that this artificial water model with an increased dipole moment would lead to an accurate description of bulk water properties. Calculation of adsorption in hydrophilic zeolites using polarizable water models will be the topic of a future study.

We investigated the influence of cation mobility and zeolite structure by calculating adsorption isotherms at $334 \mathrm{~K}$ at different simulation conditions, see Figure 6. When the cations are mobile, there is no difference in adsorption between the hydrated and the dehydrated structure. The pore volume of the two structures calculated by molecular simulation is very similar (4005 $\AA^{3} /$ unit cell for the hydrated structure, $4000 \AA^{3}$ /unit cell for the hydrated structure), and we do not expect a large difference in saturation adsorption between the two structures. The maximum difference between the atomic positions of the hydrated and dehydrated structure is $0.194 \AA$. These differences are enough to have an effect in the adsorption of water in hydrophobic structures ${ }^{11}$, but not in hydrophilic zeolites. In hydrophilic zeolites, water adsorbs preferentially coordinated to the extra framework cations, so that the influence of the detailed framework position is not so important.

The adsorption with fixed cations is lower than with mobile cations in both structures. This suggests that the mobile cations rearrange during the simulation to locations different from their initial positions, which is an often observed phenomenon in this and other zeolites ${ }^{3,52}$. The influence of cation mobility is large for the whole pressure range, which clearly shows the importance of considering cation mobility in the simulations. The adsorption with fixed and mobile cations is similar only in the dehydrated structure at low pressures. These results indicate that mobile cations at these conditions do not move far from their crystallographic positions. For example, at $1 \mathrm{~Pa}$ the maximum average displacement of a cation from its crystallographic position is $2 \AA$. Initially, there 
are four cations inside every beta cage in the dehydrated structure, and three in the hydrated structure. During the hydratation process, one of the cations inside the beta cage in de dehydrated structure migrates out of the cage. Therefore, more water molecules can be adsorbed in these cages, which are their preferential adsorption site as it was demonstrated both experimentally ${ }^{1,43}$ and by simulation $^{29}$. This migration takes place when there are between 25 and 30 water molecules adsorbed per unit cell of LTA4A (1.8 - $2.2 \mathrm{~mol} / \mathrm{kg})$.

Adsorption in the beta cages of LTA4A is limited by diffusion through 6-ring windows. Monte Carlo simulations do not have this limitation, as with this simulation method the molecules can easily overcome large free energy barriers. To establish if the force field here developed allows water to be adsorbed in the beta cages, which experimentally is the preferential adsorption site, it is necessary to perform a diffusion study by Molecular Dynamics. In this study, a LTA4A zeolite is filled with water molecules located in the alpha cage and equilibrated, checking at all times that during this process no molecules move to the beta cages. Afterwards, we run a MD simulation of at least $10 \mathrm{~ns}$, and count the number of molecules that diffuse to the beta cages. In the rigid structure no water molecule diffuses to the beta cage during the whole simulation time, while in the flexible zeolite the beta cages can easily accommodate three water molecules after only 15 ps, which enter the beta cages and leave them several times during the simulation. The explanation is that the 6-ring windows are too narrow even for the small water molecules to diffuse, and they can only cross the windows if they are allowed to breathe.

The question now is whether framework flexibility has any influence in water adsorption. It has been demonstrated that the influence of framework flexibility on the computed adsorption of small alkane molecules in zeolites is negligible ${ }^{53}$, but this has not been clearly established for water. In Fig. 6 we show the comparison between the adsorption isotherm in both rigid and flexible LTA4A, for both the hydrated and dehydrated structure with mobile cations. The flexible zeolite model only provides a slightly larger adsorption than the rigid, often within the error bars of the simulation. We can conclude that water behaves like other small molecules, and that zeolite flexibility is important for diffusion because water can only cross narrow windows when the zeolite is flexible, but it does not have much influence in adsorption.

The water adsorption siting is also consistent with experimental data, as shown in the density surface plots of Figure 7. At low pressures, water molecules first occupy the beta cages. This is due to the fact that these cages offer water a better confined environment. A few molecules also adsorb 
at the walls of the alpha cage. At higher pressures, water fills also the alpha cages, first the walls and then the center of the cage.

Finally, we test the transferability of force fields for describing water adsorption in hydrophilic zeolites. In Figure 8, we show computed water adsorption isotherms in LTA4A at $298 \mathrm{~K}$ using the force field described by Fuchs et $a l^{54}$, which perfectly reproduces water adsorption in a FAU type zeolite for different temperatures. Simulations were performed with both the Tip5pEw water model $^{37}$, and the Tip4p water model ${ }^{55}$ originally used by Fuchs et $a l .{ }^{54}$. The adsorption provided by this force field is much larger than the experimental isotherm, and the isotherm calculated with the force field developed in this work. The main reason is that the force field of Fuchs was developed to reproduce water adsorption only in the FAU zeolite. Notice also that the partial charges of the framework atoms Fuchs' model are more than twice higher than in our model. Therefore, the water-zeolite interaction is more intense and the adsorption higher. We also calculated the adsorption in a FAU-type zeolite containing 52 sodium cations per unit cell at $300 \mathrm{~K}$, and compared it with reference data ${ }^{55}$ in Figure 9. We used the characterizations of Fitch et al. ${ }^{39}$ and Olson $^{40}$ to assign positions to the atoms of the zeolite, and selected a random distribution of alumina atoms satisfying the Lowenstein's rule. The adsorption isotherms in both structures overlap. The original force field does not perform as well as in the reference ${ }^{54}$, as the calculated adsorption in FAU-type zeolites depends on the specific location of alumina atoms in the zeolite ${ }^{21}$, which is not provided in the reference data. The force field developed in this work provides a lower water adsorption in hydrophylic zeolites than the force field of Fuchs et al., resulting in a worse description of water adsorption in the FAU zeolite, but an excellent agreement with the LTA4A water isotherms.

\section{Conclusions}

The experimental water adsorption isotherms in LTA4A at different regeneration cycles show that the adsorption is always lower in the first cycle compare to successive cycles, and the same cycle and lower temperatures. This provides a clear evidence of a complete desorption at low temperatures, and a possible cation reallocation upon water adsorption. We developed a new force field for water adsorption in LTA4 that successfully reproduces experimental isotherms. The low adsorption at high pressures is attributed to the increase of the water dipole moment at conditions similar to bulk liquid water, which cannot be reproduced with our non-polarizable model. Contrarily to what happens in hydrophobic zeolites, water adsorption is not largely influenced by small changes in the zeolite structure, as in hydrophilic zeolites cations act as adsorption centers. 
Therefore, cation mobility has a large effect in adsorption: when the cations are kept fixed at their crystallographic positions, they cannot migrate to more favorable positions upon the adsorption of water, and the water adsorption is lower than when the cations are mobile. We have shown that the preferential adsorption sites in the zeolite are the beta cages, in complete agreement with experimental data. We found that water molecules can only diffuse to the beta cages when the framework model is flexible, while adsorption is not influenced by framework flexibility. Finally, we concluded that force fields developed to reproduce water adsorption in hydrophilic zeolites are in general not transferable between different zeolite types. Therefore, when studying water adsorption in hydrophilic zeolites it is necessary to develop specific force fields for specific zeolite types taking into account the particular location of alumina atoms in the structure, or more refined force fields that can account for properties such as molecular polarization. 
Table 1. Non-bonded force field parameters used in this work. $\mathrm{O}_{\mathrm{Al}}$ represent oxygen atoms of the zeolite bonded to at least one $\mathrm{Al}$ atom; $\mathrm{O}_{\mathrm{Si}}$ represent oxygen atoms of the zeolite bonded only to $\mathrm{Si}$ atoms; $\mathrm{O}_{\mathrm{w}}$, water oxygen atoms; $\mathrm{M}$ is the dummy atom in the Tip5pEw water model ${ }^{37}$. The LTA4A zeolite only contains oxygen atoms of type $\mathrm{O}_{\mathrm{Al}}$.

\begin{tabular}{lccc}
\hline Atom/s & $\boldsymbol{\varepsilon} / \mathbf{k}_{\mathbf{B}} / \mathbf{K}$ & $\boldsymbol{\sigma} / \mathbf{\AA}$ & $\mathbf{q} / \mathbf{e}$ \\
\hline $\mathrm{Si}$ & - & - & 0.786 \\
$\mathrm{Al}$ & - & - & 0.486 \\
$\mathrm{O}_{\mathrm{Al}}$ & - & - & -0.414 \\
$\mathrm{O}_{\mathrm{Si}}$ & - & - & -0.393 \\
$\mathrm{Na}$ & 251.780 & 3.1440 & 0.383 \\
$\mathrm{O}_{\mathrm{w}}$ & 89.516 & 3.0970 & - \\
$\mathrm{H}$ & - & - & 0.241 \\
$\mathrm{M}$ & - & - & -0.241 \\
$\mathrm{O}_{\mathrm{Al}}-\mathrm{Na}$ & 33.000 & 3.2000 & \\
$\mathrm{O}_{\mathrm{Al}}-\mathrm{O}_{\mathrm{w}}$ & 13.710 & 3.3765 & \\
$\mathrm{O}_{\mathrm{Si}}-\mathrm{Na}_{\mathrm{Na}}$ & 33.000 & 3.2000 & \\
$\mathrm{O}_{\mathrm{Si}}-\mathrm{O}_{\mathrm{w}}$ & 13.710 & 3.3765 & \\
$\mathrm{Na}-\mathrm{O}_{\mathrm{w}}$ & 75.000 & 2.3900 &
\end{tabular}


Figure 1. Structure of the dehydrated LTA4A zeolite ${ }^{30}$. Yellow, silicon atoms; green, alumina atoms; red, oxygen atoms. The initial cation adsorption sites are marked with circles. This figure will appear in color in the electronic version of this article.

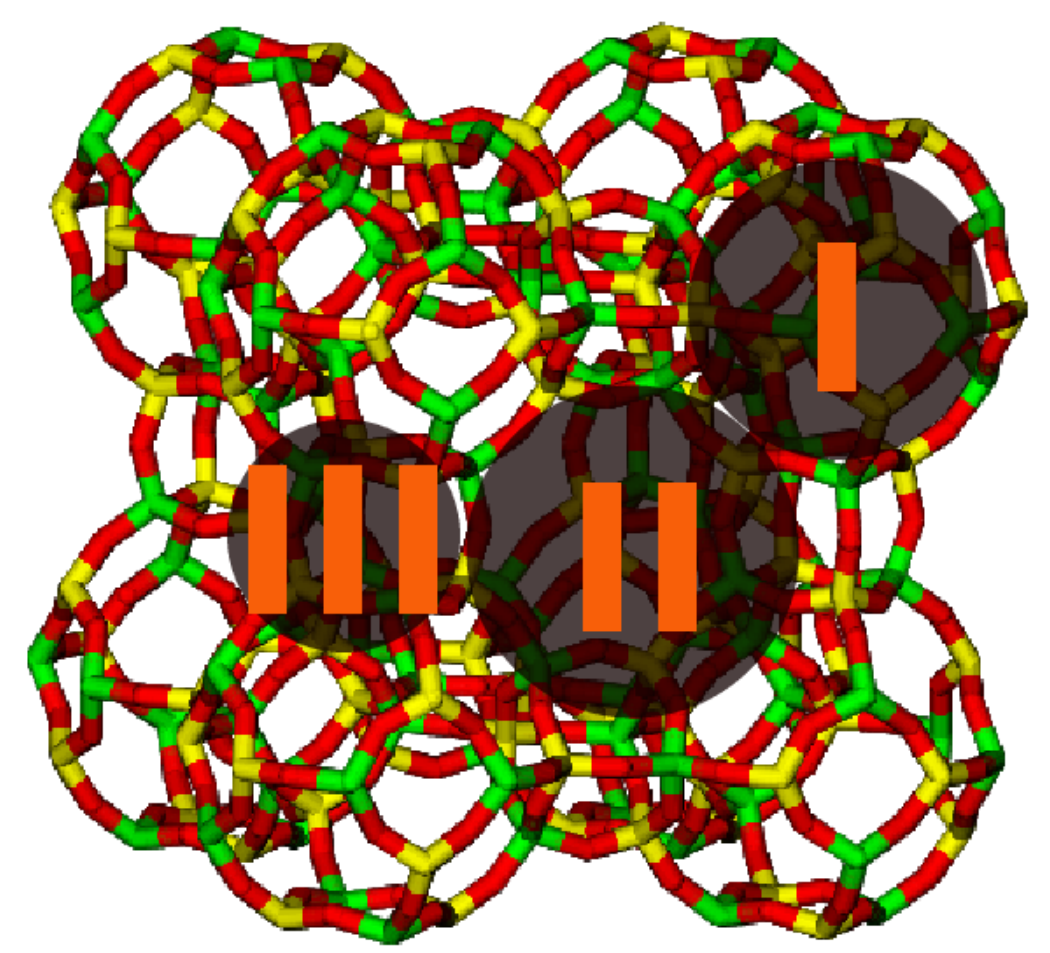


Figure 2. Experimental adsorption isotherms of water in LTA4A at $334 \mathrm{~K}$. Left column: comparative of different cycles after degassing at (a) $423 \mathrm{~K}$, (b) $523 \mathrm{~K}$ and (c) $723 \mathrm{~K}$; Closed symbols, first adsorption cycle; open symbols, second adsorption cycle; crossed symbols, third adsorption cycle; Right column: comparative of the degassing temperature for the (d) first, (e) second and (f) third cycle; squares, degassing temperature of $423 \mathrm{~K}$; triangles, degassing temperature of $523 \mathrm{~K}$; diamonds, degassing temperature of $573 \mathrm{~K}$.
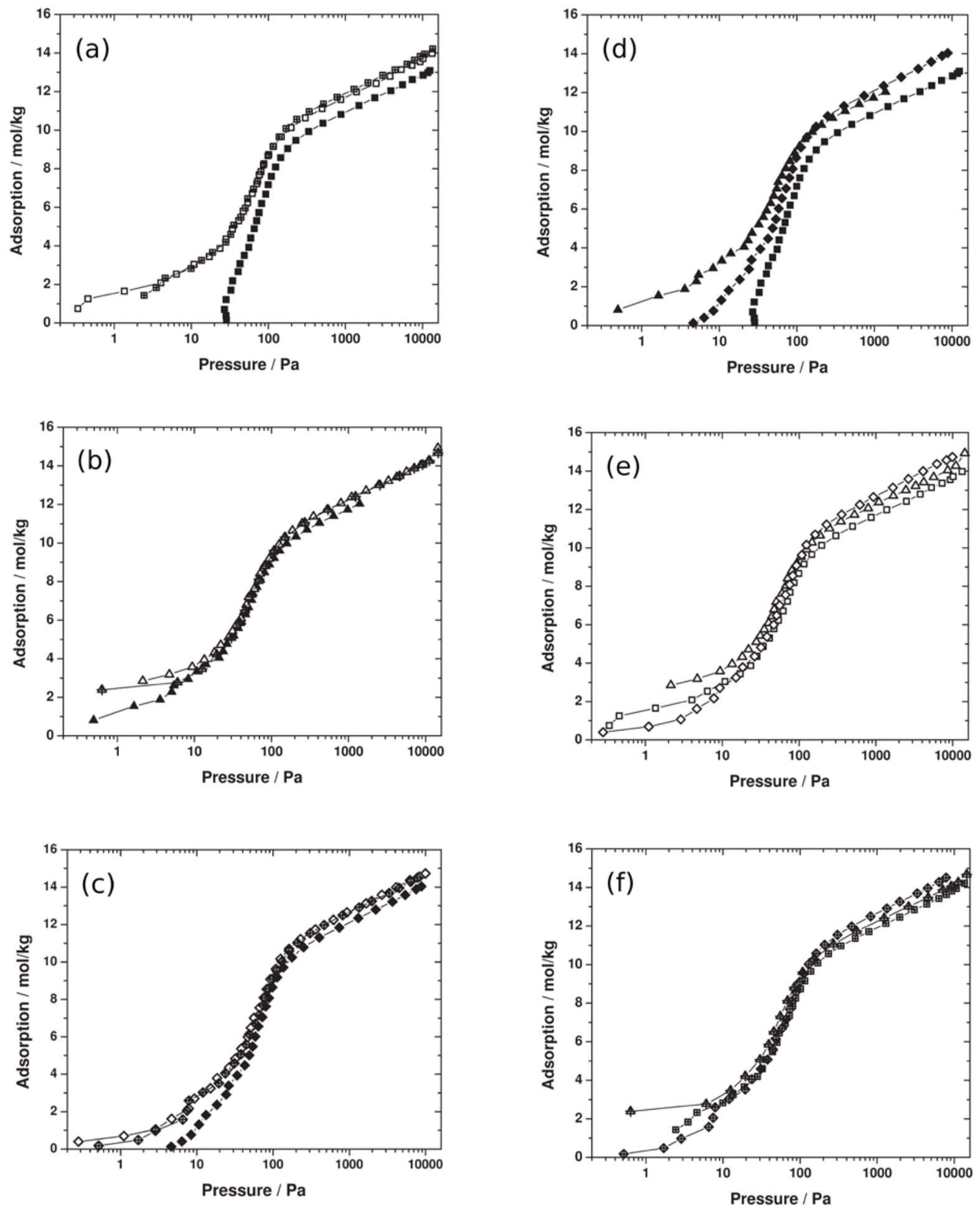
Figure 3. Experimental adsorption isotherms of water in LTA4A at temperatures close to $298 \mathrm{~K}$. Squares, this work (298 K); circles, data of Gorbach et al. ${ }^{9}$ (298 K); triangles, data of Morris ${ }^{46}$ (298 $\mathrm{K})$; triangles down, data of Okamoto et al. ${ }^{8}$ (298 K); diamonds, data of Valiullin et al. ${ }^{47}$ (300 K).

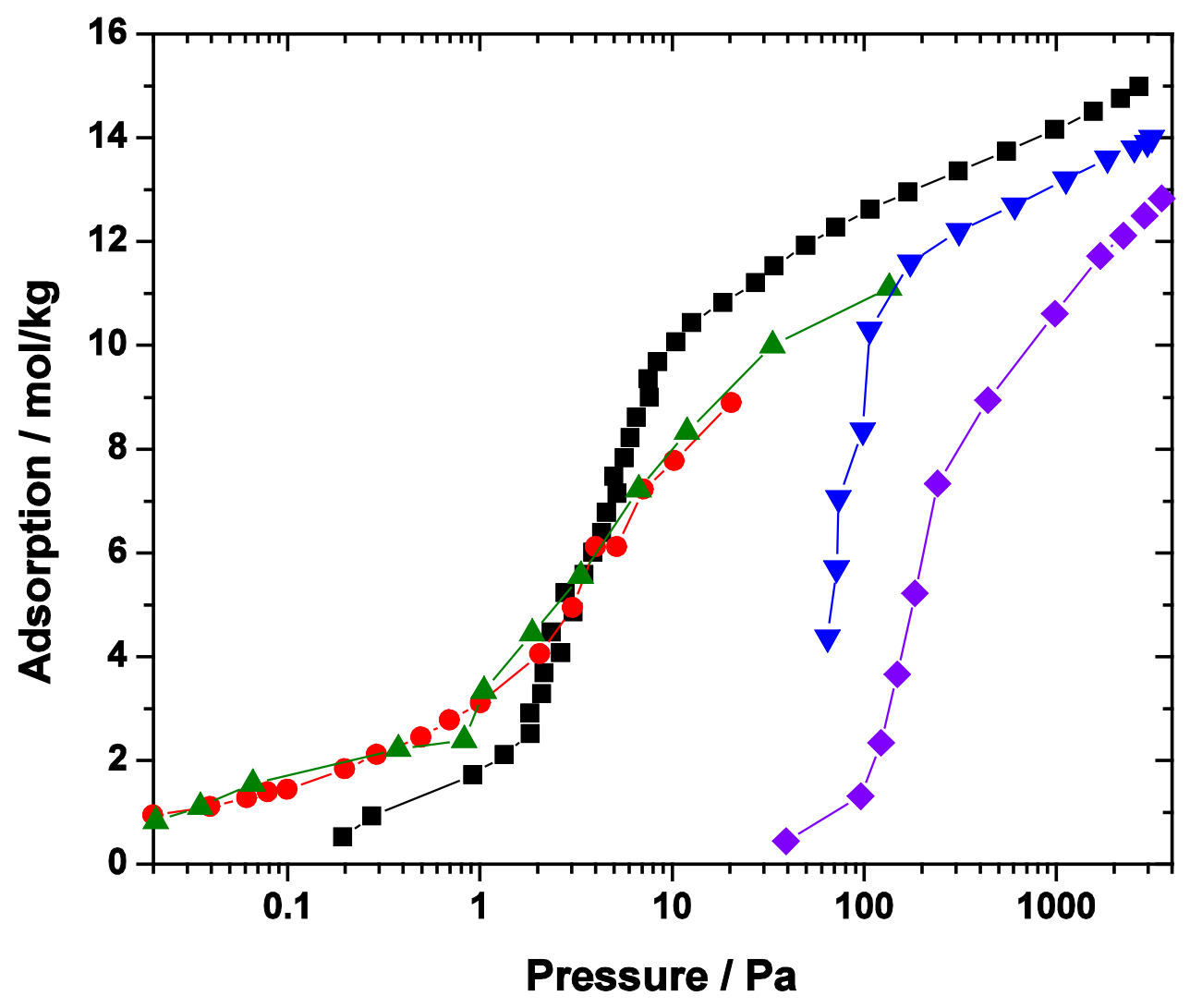


Figure 4. Experimental adsorption isotherms of water in LTA4A at temperatures close to $334 \mathrm{~K}$. Squares, this work (334 K); circles, data of Gorbach et al. ${ }^{9}$ (334 K); triangles, data of Morris ${ }^{46}$ (338 $\mathrm{K})$; triangles down, data of Pera-Titus et al. ${ }^{48}$ (333 K).

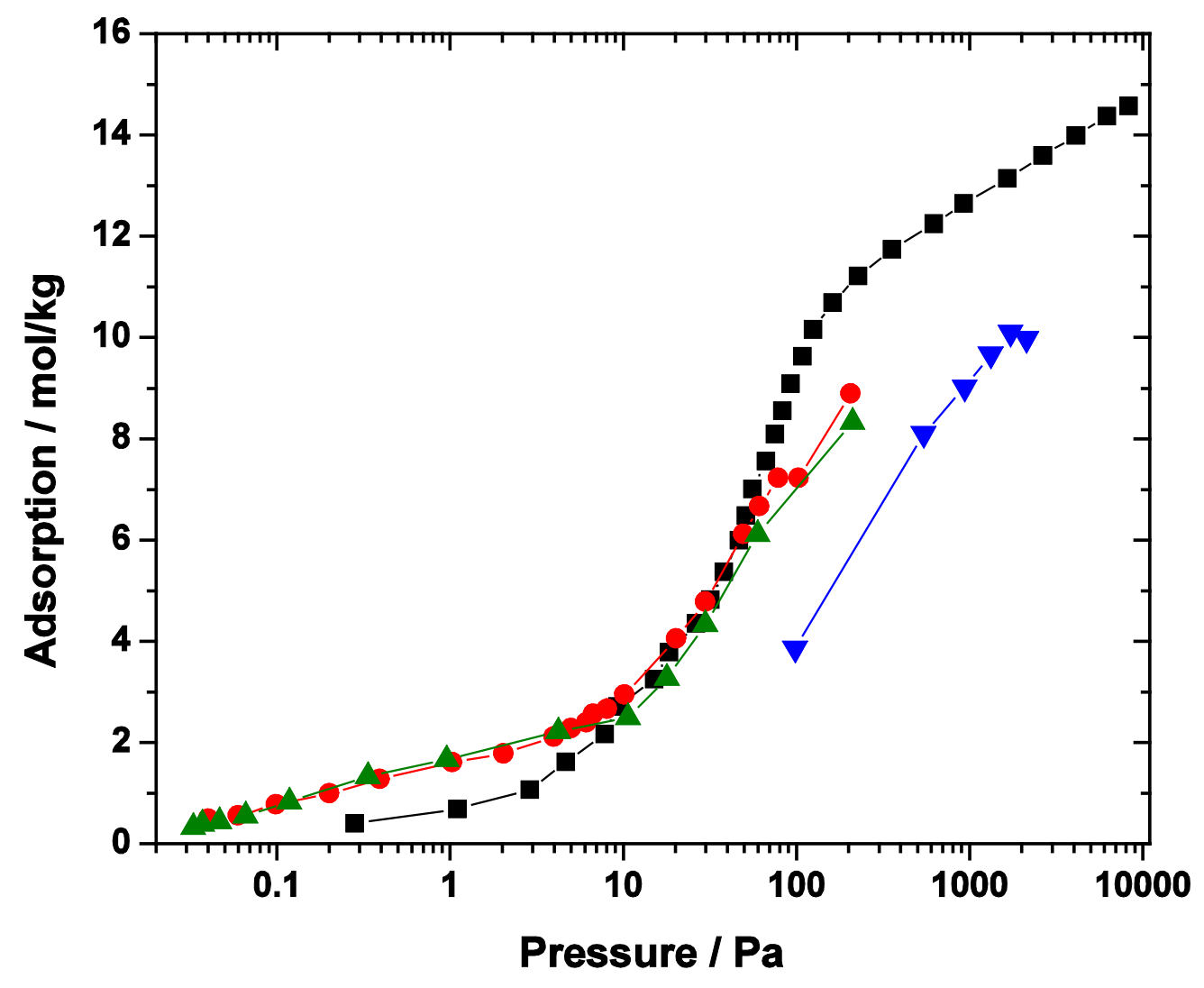


Figure 5. Adsorption isotherms of water in LTA4A calculated with the fitted force field parameters (open symbols) in the hydrated structure, and compared with experimental data (closed symbols), at different temperatures: squares, $273 \mathrm{~K}$ (experimental data from Gorbach et al. ${ }^{9}$ ); circles, $298 \mathrm{~K}$; triangles, $334 \mathrm{~K}$; diamonds, $374 \mathrm{~K}$ (experimental data from Grobach et al. ${ }^{9}$ ). Error bars are within the symbol size.

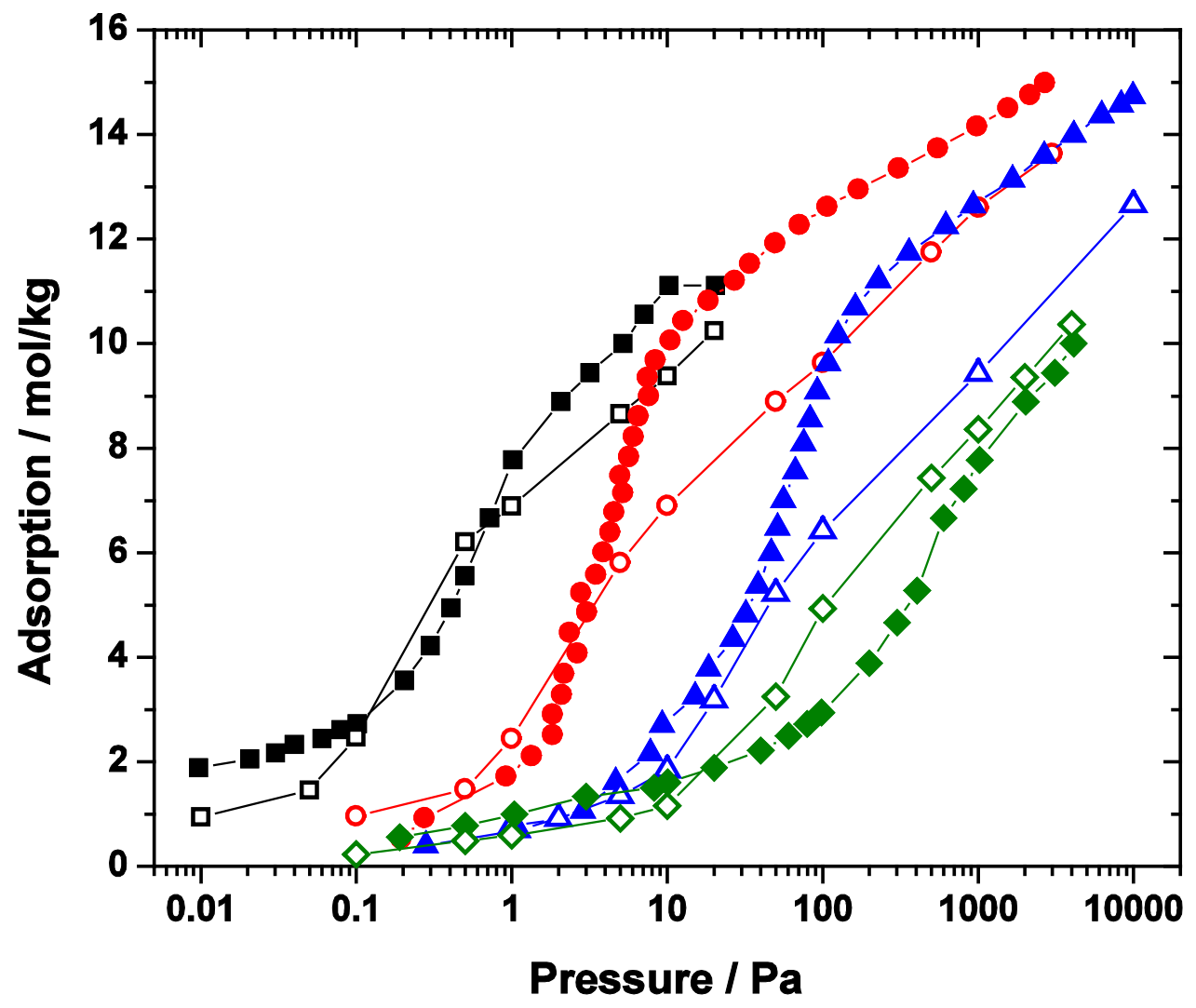


Figure 6. Adsorption isotherms of water in LTA4A at $334 \mathrm{~K}$, calculated with different simulation parameters. Squares, experimental data; circles, hydrated structure; triangles, dehydrated structure; closed symbols, mobile cations; open symbols, fixed cations; crossed symbols, flexible structure with mobile cations; asterisk, hydrated structure with mobile cations, and a water dipole moment of 2.5 D. Error bars are within the symbol size.

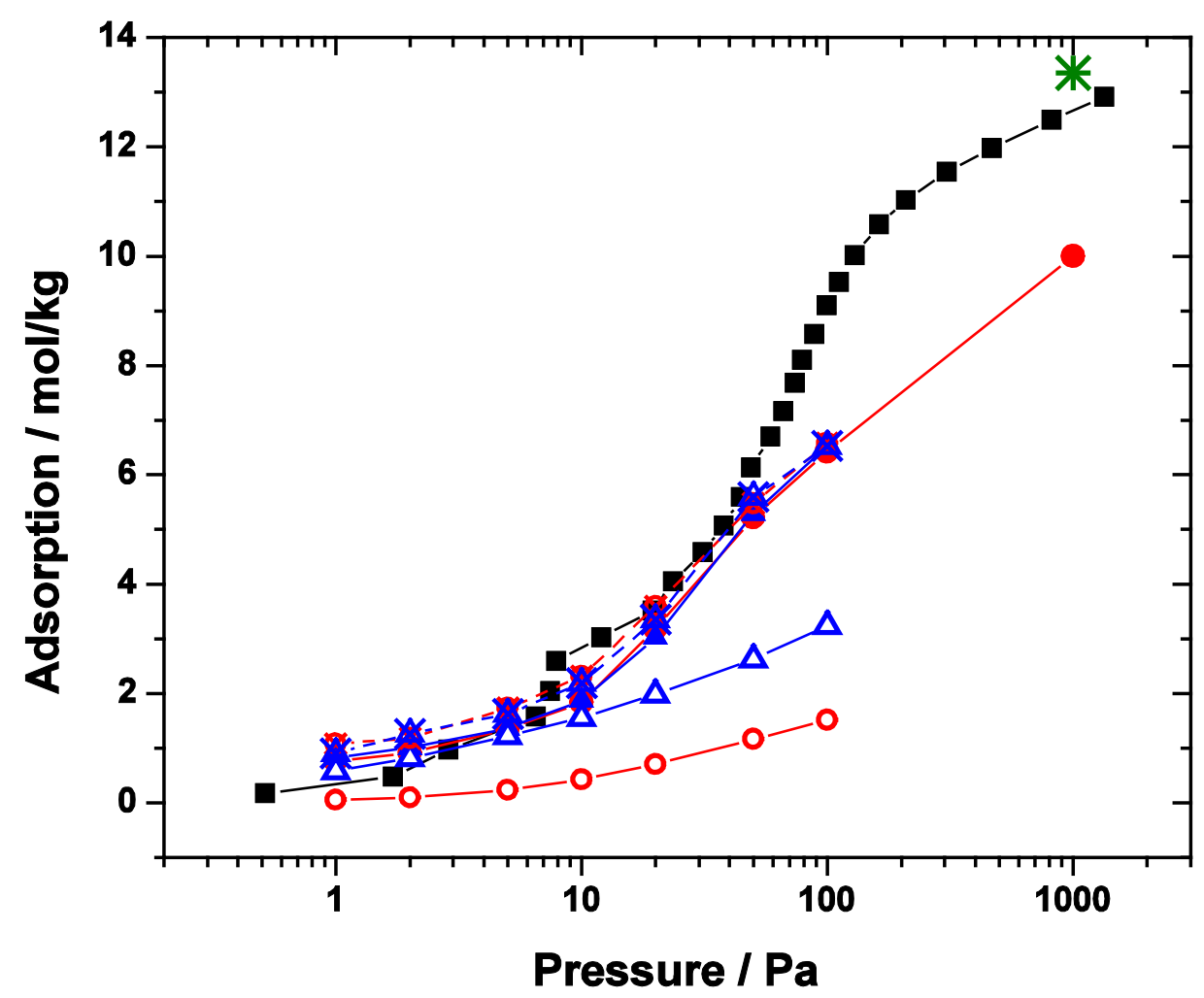


Figure 7. Water density plots in the hydrated LTA4A structure at $334 \mathrm{~K}$ and different pressures of water in the vapor phase. Top, $1 \mathrm{~Pa}$; bottom, 100Pa.
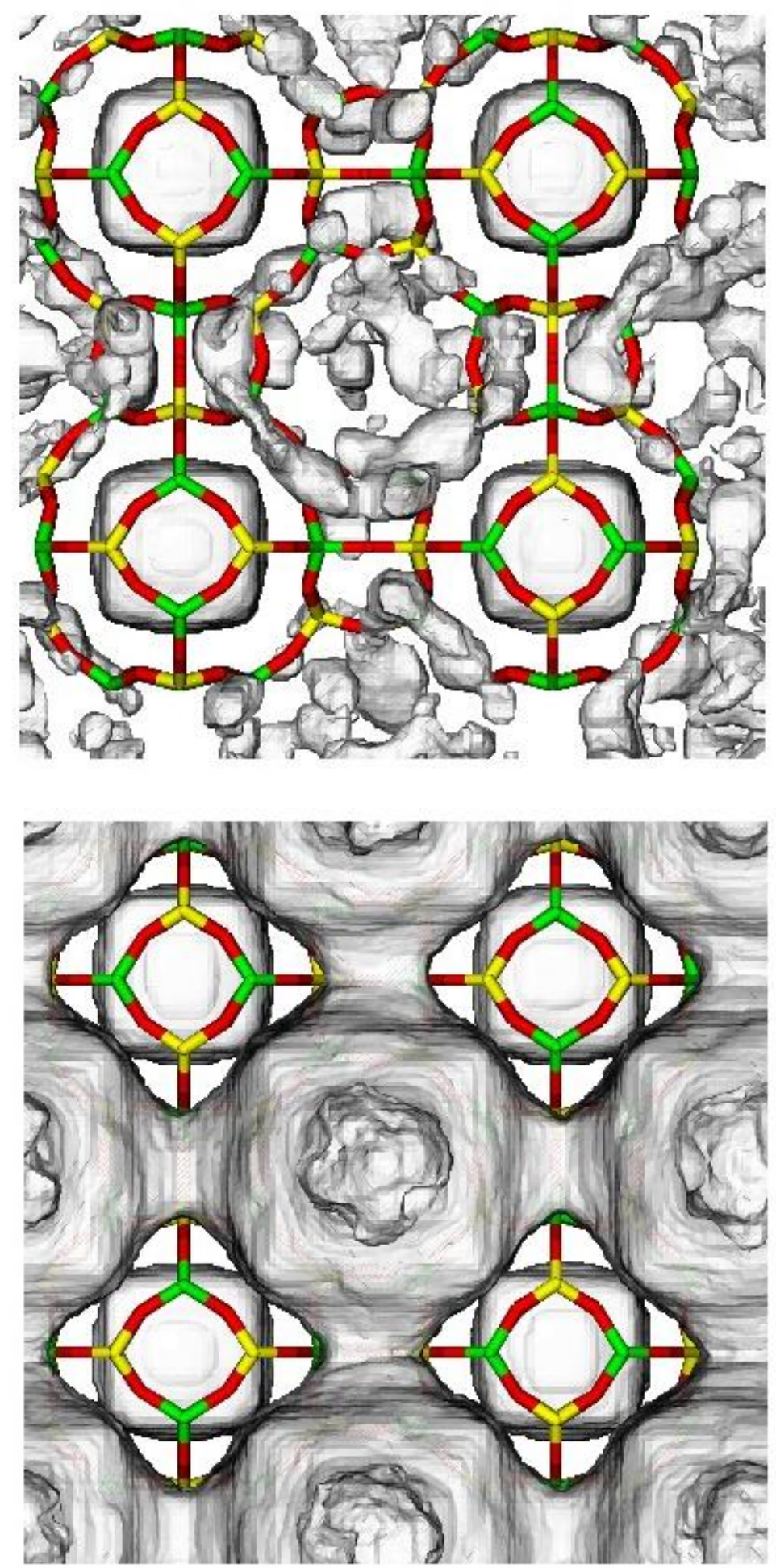
Figure 8. Adsorption isotherms of water in the hydrated structure of LTA4A at $298 \mathrm{~K}$. Squares, experimental data; triangles, simulations using the force field developed in this work; diamonds, simulations using the force field by Fuchs et $a l^{54}$ and the Tip5pEw ${ }^{37}$ water model; triangles down, simulations using the force field by Fuchs et $a l^{54}$ and the Tip4p water model ${ }^{55}$. Cations are mobile in the simulations. Error bars are within the symbol size.

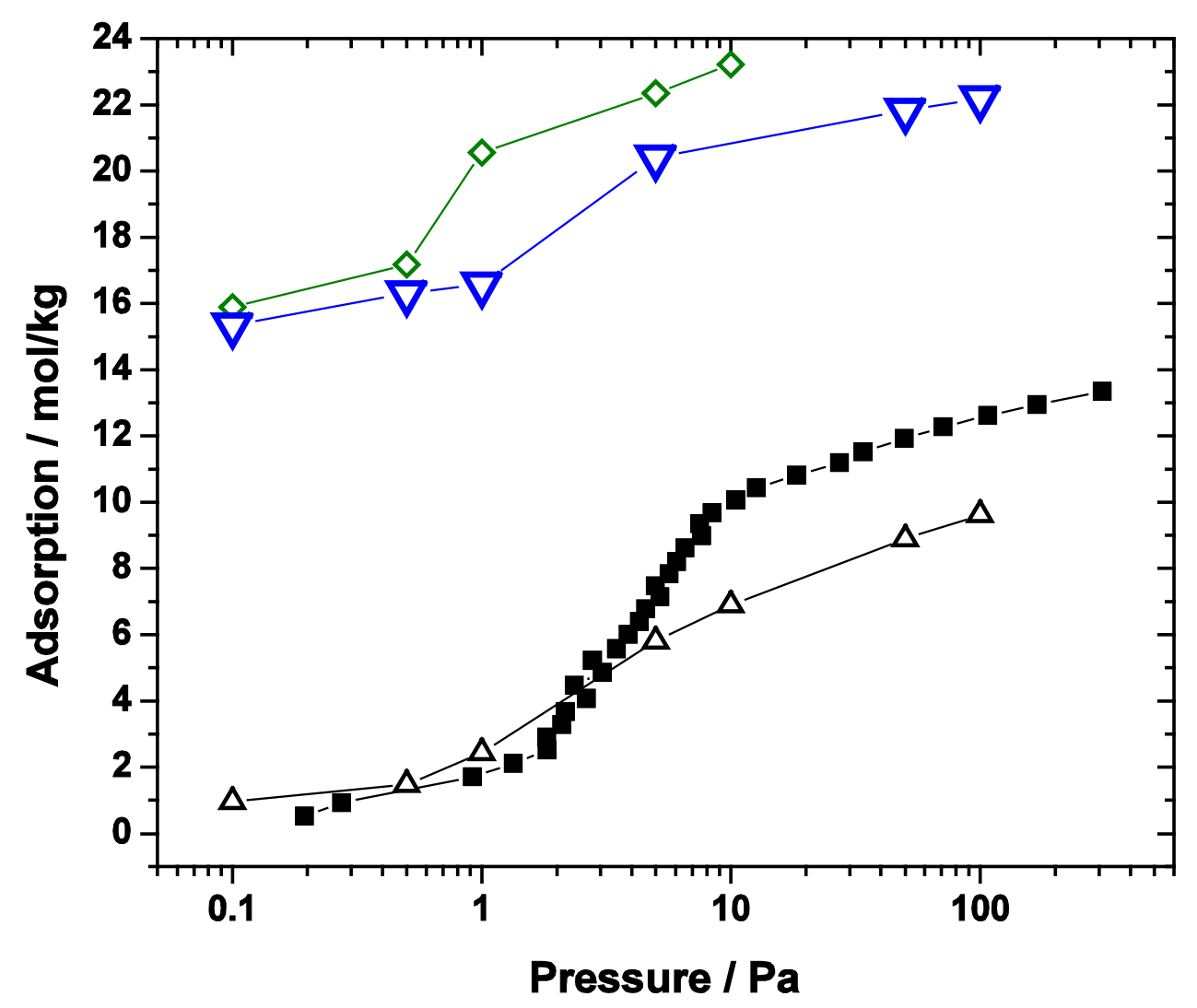


Figure 9. Adsorption isotherms of water in FAU containing 52 sodium cations per unit cell at 300 $\mathrm{K}$, calculated using different simulation parameters. Squares, experimental data ${ }^{55}$; triangles,

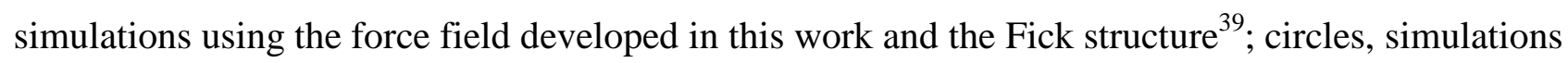
using the force field developed in this work and the Olson structure ${ }^{40}$; diamonds, simulations using the force field by Fuchs et $a l^{54}$ (the water model used is Tip4 $\mathrm{p}^{55}$ ). Error bars are within the symbol size.

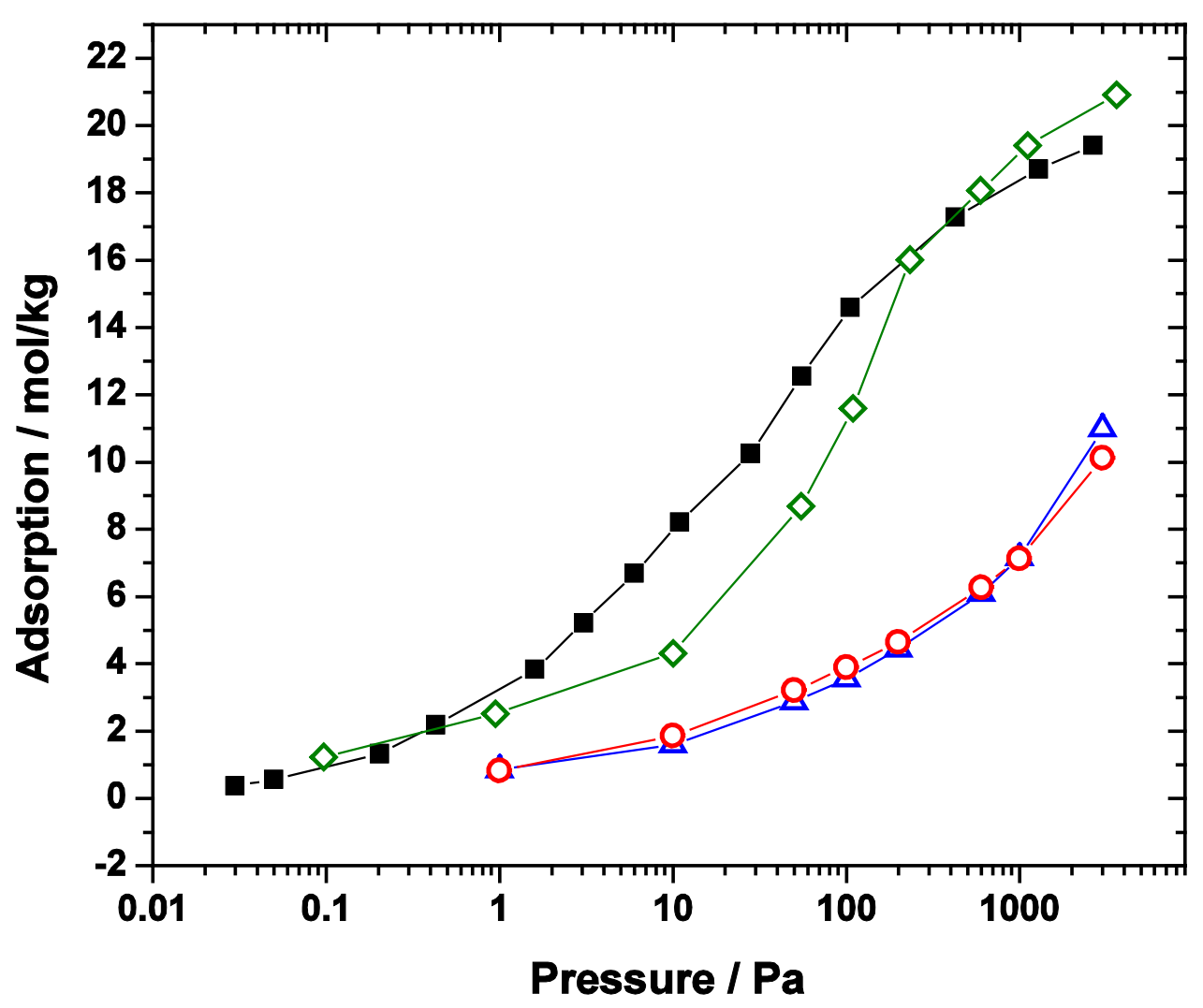




\section{Acknowledgements}

The authors would like to acknowledge A. Silvestre-Albero and E. Gadea for their help with the water adsorption measurements, the European Research Council through an ERC Staring Grant (Sofia Calero), and the MICINN (CTQ2010-16077) project.

\section{References}

(1) Zhu, W.; Gora, L.; van den Berg, A. W. C.; Kapteijn, F.; Jansen, J. C.; Moulijn, J. A. Journal of Membrane Science 2005, 253, 57.

(2) Huang, A.; Yang, W.; Liu, J. Separation and Purification Technology 2007, 56, 158.

(3) Kyotani, T.; Ikeda, T.; Saito, J.; Nakane, T.; Hanaoka, T.; Mizukami, F. Industrial \& Engineering Chemistry Research 2009, 48, 10870.

(4) Li, Y. S.; Chen, H. L.; Liu, J.; Li, H. B.; Yang, W. S. Separation and Purification Technology 2007, 57, 140.

(5) Liang, H.; Gao, H.; Kong, Q.; Chen, Z. Journal of Chemical and Engineering Data 2007, 52, 695.

(6) Camargo Fernandes-Machado, N. R.; Malachini Miotto-Bigatao, D. M. Quimica Nova 2007, 30, 1108.

(7) Adell, A.; Petrissans, J. Talanta 1998, 45, 777.

(8) Okamoto, K.; Kita, H.; Horii, K.; Tanaka, K.; Kondo, M. Industrial \& Engineering Chemistry Research 2001, 40, 163.

(9) Gorbach, A.; Stegmaier, M.; Eigenberger, G. Adsorption-Journal of the International Adsorption Society 2004, 10, 29.

(10) Liu, B.; Garcia-Perez, E.; Dubbeldam, D.; Smit, B.; Calero, S. Journal of Physical Chemistry C 2007, 111, 10419.

(11) Castillo, J. M.; Dubbeldam, D.; Vlugt, T. J. H.; Smit, B.; Calero, S. Molecular Simulation 2009, 35, 1067.

(12) Bellat, J. P.; Paulin, C.; Jeffroy, M.; Boutin, A.; Paillaud, J. L.; Patarin, J.; Di Lella, A.; Fuchs, A. Journal of Physical Chemistry C 2009, 113, 8287.

(13) Abrioux, C.; Coasne, B.; Maurin, G.; Henn, F.; Boutin, A.; Di Lella, A.; NietoDraghi, C.; Fuchs, A. H. Adsorption-Journal of the International Adsorption Society 2008, 14, 743. 22, 270.

(14) Guo, X. D.; Huang, S. P.; Teng, J. W.; Xie, Z. K. Acta Physico-Chimica Sinica 2006,

(15) Yazaydin, A. O.; Thompson, R. W. Microporous and Mesoporous Materials 2009, 123, 169.

(16) Channon, Y. M.; Catlow, C. R. A.; Gorman, A. M.; Jackson, R. A. Journal of Physical Chemistry B 1998, 102, 4045.

(17) Ruiz-Salvador, A. R.; Almora-Barrios, N.; Gomez, A.; Lewis, D. W. Physical Chemistry Chemical Physics 2007, 9, 521.

(18) Maurin, G.; Bell, R. G.; Devautour, S.; Henn, F.; Giuntini, J. C. Journal of Physical Chemistry B 2004, 108, 3739.

(19) Coudert, F.-X.; Cailliez, F.; Vuilleumier, R.; Fuchs, A. H.; Boutin, A. Faraday Discussions 2009, 141, 377.

(20) Di Lella, A.; Desbiens, N.; Boutin, A.; Demachy, I.; Ungerer, P.; Bellat, J. P.; Fuchs, A. H. Physical Chemistry Chemical Physics 2006, 8, 5396.

(21) Garcia-Perez, E.; Dubbeldam, D.; Liu, B.; Smit, B.; Calero, S. Angewandte ChemieInternational Edition 2007, 46, 276. 
(22) Furukawa, S.; Goda, K.; Zhang, Y.; Nitta, T. Journal of Chemical Engineering of Japan 2004, 37, 67.

(23) Jaramillo, E.; Chandross, M. Journal of Physical Chemistry B 2004, 108, 20155.

(24) Kristof, T.; Csanyi, E.; Rutkai, G.; Merenyi, L. Molecular Simulation 2006, 32, 869.

(25) Wu, J. Y.; Liu, Q. L.; Xiong, Y.; Zhu, A. M.; Chen, Y. Journal of Physical Chemistry B 2009, 113, 4267.

(26) Faux, D. A.; Smith, W.; Forester, T. R. Journal of Physical Chemistry B 1997, 101, 1762.

(27) Gren, W.; Parker, S. C.; Slater, B.; Lewis, D. W. Journal of Physical Chemistry C, $114,9739$.

(28) Allen, J. P.; Gren, W.; Molinari, M.; Arrouvel, C.; Maglia, F.; Parker, S. C. Molecular Simulation 2009, 35, 584.

(29) Higgins, F. M.; de Leeuw, N. H.; Parker, S. C. Journal of Materials Chemistry 2002, 12, 124.

(30) Pluth, J. J.; Smith, J. V. Journal of the American Chemical Society 1980, 102, 4704.

(31) Gramlich, V.; Meier, W. M. Zeitschrift Fur Kristallographie Kristallgeometrie Kristallphysik Kristallchemie 1971, 133, 134.

(32) Pathria, R. K. Statistical Mechanics, Second ed.; Butterworth-Heinemann: Oxford, 1996; Vol. 1.

(33) Siepmann, J. I.; Frenkel, D. Molecular Physics 1992, 75, 59.

(34) Calero, S.; Dubbeldam, D.; Krishna, R.; Smit, B.; Vlugt, T. J. H.; Denayer, J. F. M.; Martens, J. A.; Maesen, T. L. M. Journal of the American Chemical Society 2004, 126, 11377.

1102.

(35) Vlugt, T. J. H.; Krishna, R.; Smit, B. Journal of Physical Chemistry B 1999, 103,

(36) Frenkel, D.; Smit, B. Understanding Molecular Simulations: From Algorithms to Applications, 2nd ed.; Academic Press: San Diego, 2002.

(37) Rick, S. Journal of Chemical Physics 2004, 120, 6085.

(38) Garcia-Sanchez, A.; Ania, C. O.; Parra, J. B.; Dubbeldam, D.; Vlugt, T. J. H.; Krishna, R.; Calero, S. Journal of Physical Chemistry C 2009, 113, 8814.

(39) Fitch, A. N.; Jobic, H.; Renouprez, A. Journal of Physical Chemistry 1986, 90, 1311.

(40) Olson, D. H. Zeolites 1995, 15, 439.

(41) Nicholas, J. B.; Hopfinger, A. J.; Trouw, F. R.; Iton, L. E. Journal of the American Chemical Society 1991, 113, 4792.

(42) Allen, M. P.; Tildesley, D. J. Computer Simulations of Liquids; Clarendon Press: Oxford, 1987.

(43) Vucelic, V.; Vucelic, D.; Karaulic, D.; Susic, M. Thermochimica Acta 1973, 7, 77.

(44) Goryainov, S. V.; Secco, R. A.; Huang, Y.; Liu, H. Physica B-Condensed Matter 2007, 390, 356.

(45) Ohgushi, T.; Ishimaru, K.; Adachi, Y. Journal of Physical Chemistry C 2009, 113, 2468.

(46) Morris, B. Journal of Colloid and Interface Science 1968, 28, 149.

(47) Valiullin, R.; Kaerger, J.; Cho, K.; Choi, M.; Ryoo, R. Microporous and Mesoporous Materials 2011, 142, 236.

(48) Pera-Titus, M.; Fite, C.; Sebastian, V.; Lorente, E.; Llorens, J.; Cunill, F. Industrial \& Engineering Chemistry Research 2008, 47, 3213.

(49) Nelder, J. A.; Mead, R. Computer Journal 1965, 7, 308.

(50) Simonot-Grange, M. H.; Belhamidi-El Hannouni, F. Thermochimica Acta 1984, 77,

311.

(51) Halasz, I.; Kim, S.; Marcus, B. Molecular Physics 2002, 100, 3123.

(52) Beauvais, C.; Boutin, A.; Fuchs, A. H. Comptes Rendus Chimie 2005, 8, 485.

(53) Vlugt, T. J. H.; Schenk, M. Journal of Physical Chemistry B 2002, 106, 12757. 
(54) Fuchs, A. H.; Boutin, A.; Teuler, J. M.; Di Lella, A.; Wender, A.; Tavitian, B.; Ungerer, P. Oil \& Gas Science and Technology-Revue D Ifp Energies Nouvelles 2006, 61, 571.

(55) Jorgensen, W. L.; Chandrasekhar, J.; Madura, J. D.; Impey, R. W.; Klein, M. L. Journal of Chemical Physics 1983, 79, 926. 